\title{
Periodic forcing in a three-level cellular automata model for a vector-transmitted disease
}

\author{
L. B. L. Santos, M. C. Costa, S. T. R. Pinho, * and R. F. S. Andrade \\ Instituto de Física, Universidade Federal da Bahia, 40210-340 Salvador, Brazil \\ F. R. Barreto, M. G. Teixeira, and M. L. Barreto \\ Instituto de Saúde Coletiva, Universidade Federal da Bahia, 40110-140 Salvador, Brazil \\ (Received 1 October 2008; revised manuscript received 14 March 2009; published 6 July 2009)
}

\begin{abstract}
A periodically forced two-dimensional cellular automata model is used to reproduce and analyze the complex spatiotemporal patterns observed in the transmission of vector infectious diseases. The system, which comprises three population levels, is introduced to describe complex features of the dynamics of the vectortransmitted dengue epidemics, known to be very sensitive to seasonal variables. The three coupled levels represent the human, the adult, and immature vector populations. The dynamics includes external seasonality forcing, human and mosquito mobility, and vector control effects. The model parameters, even if bounded to well-defined intervals obtained from reported data, can be selected to reproduce specific epidemic outbursts. In the current study, explicit results are obtained by comparison with actual data retrieved from the time series of dengue epidemics in two cities in Brazil. The results show fluctuations that are not captured by mean-field models. It also reveals the qualitative behavior of the spatiotemporal patterns of the epidemics. In the extreme situation of the absence of external periodic drive, the model predicts a completely distinct long-time evolution. The model is robust in the sense that it is able to reproduce the time series of dengue epidemics of different cities, provided that the forcing term takes into account the local rainfall modulation. Finally, an analysis is provided of the effect of the dependence between epidemics threshold and vector control actions, both in the presence and absence of human mobility factor.
\end{abstract}

DOI: 10.1103/PhysRevE.80.016102

PACS number(s): 89.75.Fb, 87.18.-h, 87.16.aj, 87.19.xd

\section{INTRODUCTION}

Understanding the rather complex dynamics of transmissible diseases is of utmost importance for improving life quality and even warranting the survival of some human population groups. To achieve this task, interdisciplinary efforts are necessary. They certainly include the use of recently developed techniques to study complex systems [1] that have been mostly carried out by physicists and mathematicians, also in the cases of biologically motivated problems [2,3]. At the beginning of the 21 st century, both directly transmitted diseases, such as tuberculosis and acquired immunodeficiency syndrome (AIDS), as well as vector-transmitted diseases, such as dengue and malaria, are still not controlled. The intense flux of people both at global level and within large cities [4] increases the complexity of transmitted disease propagation [5]. For vector-transmitted diseases, there are already indications that climatic conditions and vector mobility may increase the number of cases [6]. In the case of dengue, an arboviral disease transmitted to humans by Aedes mosquitoes (mainly Aedes Aegypti), several factors favoring transmission are found in large tropical and subtropical urban centers [7]: human concentration, large intercity and intracity human mobilities, and climatic conditions for the vector proliferation (high humidity and temperature between $15^{\circ} \mathrm{C}$ and $40^{\circ} \mathrm{C}$ ). Further, dengue outbursts are quite sensitive to seasonal variations in pluviometric precipitations, humidity, and temperature. The disease, which may be caused by four different virus serotype (DenV1-DenV4), reaches yearly

\footnotetext{
*Corresponding author; suani@ufba.br
}

some 50 million people in more than 60 countries, with $\sim 21000$ casualties [8].

Since 1992 [9], ordinary differential equation (ODE) models have been proposed to analyze dengue interhost dynamics and the effect of vector control actions. More recently, several attempts to introduce spatial dependence on the disease propagation have been reported. They are based on partial differential equation [10] and cellular automata (CA) [11] models, as well as on data analysis techniques [12]. In Ref. [11], the authors proposed a model that takes into account only the description of mosquito population, which may be found in the adult and immature phases. However, a more accurate description of the dengue propagation must include, besides the interaction among humans and mosquitoes, the vector and human mobility, the effect of control actions, and an explicit climatic periodic forcing on the mosquito immature phase. To our knowledge, no previous investigation has taken into account all of these factors.

In order to appropriately model complex systems, the elements of which belong to different classes, a multilevel CA approach seems quite suitable. In this work, we investigate an interhost three-level CA model, which describes the pertinent population groups in an urban environment: human, adult vector (mosquito), and immature vector in the aquatic phase. As we will detail later on, the model includes all of the quoted effects: external forcing to describe the environment influence on the vector life cycle, interaction between different groups, and new terms to describe the effect of human and vector mobility and control actions. The results provided by the model reproduce actual time series from two well-documented dengue epidemics in two Brazilian urban centers $[13,14]$ and qualitatively agree with some main features of observed spatiotemporal transmission patterns. This 
is one major advantage of using the CA approach, which is able to capture relevant features of spatiotemporal patterns observed in transmitted diseases such as dengue [15]. We also show that in the absence of a periodic forcing, the actual epidemic outbursts are not reproduced, supporting the claims of the importance of climatic aspects to trigger local events. As the model describes the behavior of the exposed population for larger time intervals under the presence of climatic seasonal variations, it is possible to follow the effect of vector control actions. Our results provide insights on the quantitative dependence between the epidemic threshold and the parameter describing the intensity of vector control.

The current investigation goes along several successful works to describe the dynamics of disease propagation based on CA intrahost models (for instance, AIDS, [16], malaria [17], and cancer [18]) as well as on interhost models [19]. It is also worth mentioning that a CA epidemic model, with multiple interacting levels, has been explored in alternative topologies as complex networks. There, nodes represent patches of regular lattices [20] submitted to a contact process dynamics [21].

This paper is organized as follows: in Sec. II, we introduce the CA local rules, comparing them to other models in the literature. Section III discusses the choice of parameter values in our simulations. In Sec. IV, we present our results, comparing them with actual data: the simulated time series (Sec. IV A) resulting from the periodic forcing seasonal effects, the simulated spatiotemporal patterns, and the vector control associated to human mobility effect (Sec. IV B). Finally, Sec. V closes this paper with concluding remarks and perspectives.

\section{MODEL}

The CA model consists of three two-dimensional square lattices (levels), each one with $N_{s}=L \times L$ sites. The CA is subjected to closed boundary conditions in order to mimic the disease spreading in a city. In the distinct levels, each site describes the respective local populations: human $(H)$, mosquito $(M)$, and immature vector in the aquatic phase $(A)$. Humans follow susceptible, exposed, infectious, and removed (SEIR) dynamics while mosquitoes, which usually die before being removed, follow a simpler threecompartment SEI version [9]. The CA interlayer interaction rules locally couple the three involved levels by means of the usual rate equation description. They consider interactions between $H$ and $M$ levels, and the $A$ to $M$ vector population flux. The CA Moore neighborhood with radius 1 allows, for each site of a given layer, a maximum of nine neighbors in the level it interacts with (see Fig. 1). If we compare the results to actual data, each neighborhood corresponds to a set of distinct spatial units (census tracts) into which the reported cases are assigned to.

We restrict ourselves to the one-serotype situation, although the model can be extended to simulate the dynamics of transmission with more than one serotype, considering permanent immunity with respect to each homolog. Such strategy seems indicated in a first investigation, since it provides a validation of the model with the minimum required

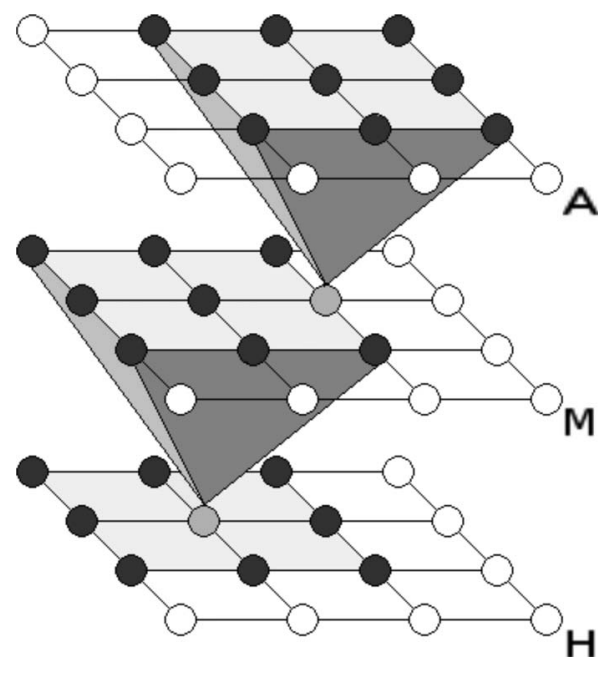

FIG. 1. Diagram of different lattices: aquatic phase of the vector $(A)$, mosquito $(M)$, and human $(H)$. Note that each element of the $H$ and $A$ lattices "sees" up to nine neighbors of the $M$ lattice (and vice versa).

number of parameters and CA states. As it will become clear, even this simplified model requires a large number of parameters for the adequate description of the many different factors influencing the dynamics of the system.

The CA sites of the $A$ (aquatic vector) level can be found in four different states: egg $(E)$, larvae $(L)$, pupae $(P)$, and breeding $(B)$. The sites of the $M$ (mosquito) level are allowed to assume three distinct states (SEI compartments): susceptible $(S M)$, exposed $(E M)$, and infectious $(I M)$. Finally, four states are available for the sites of the $H$ (human) level: susceptible $(S H)$, exposed $(E H)$, infectious $(I H)$, and recovered $(R H)$. Moreover, the sites of $A$ and $M$ levels can be in empty states, denoted by $E A S$ and $E M S$. The local interaction rules, based on the entomological [22] and epidemiological aspects [23], are as follows (see Fig. 2):

$A$ level: $E, L, P$, and $B$ states evolve from the preceding one after the $E$ eclosion period $t_{e}, L$ phase period $t_{l}$, and $P$ phase period $t_{p}$. An empty site $E A S$ may be replaced with probability $f_{S}(t)$ by an $E$ state if there is at least one occupied site in its $M$ level Moore neighborhood. The transition from $E$ to $L$ compartments also depends on $f_{s}(t)$, much as the

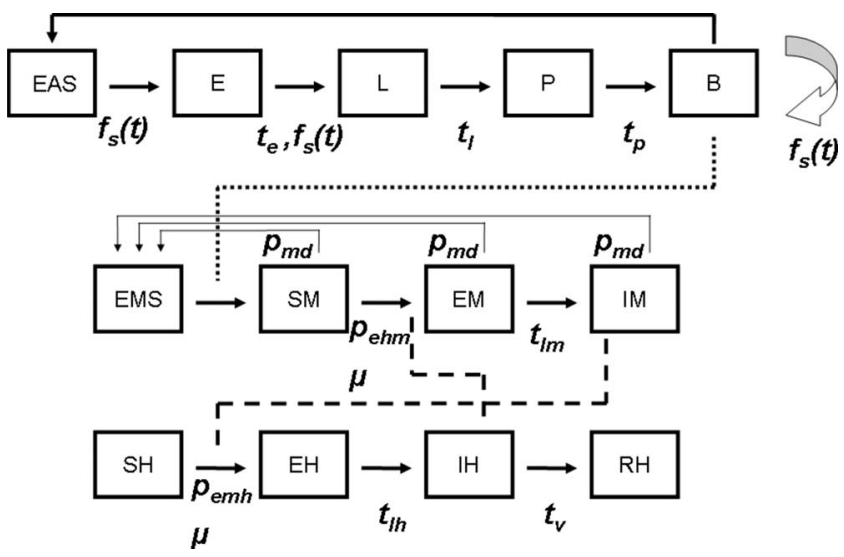

FIG. 2. A schematic of the local rules of the model. 
persistence of $B$, which releases an adult mosquito $S M$ to a $E A S$ site of the $M$ level.

$M$ level: the population in the $M$ level results from the dynamics in $A$ phase. Adult population $M$ dies according to a death probability $p_{m d}$ in any state. The transition from a $S M$ state into $E M$ depends on the number of neighbor sites in the $H$ level that occupy the $I H$ state, on the local effective mosquito-human biting probability $p_{e h m}$, and on the human mobility $\mu$. A site in the $E M$ state evolves to $I M$ after the $M$ virus latent period $t_{l m}$.

$H$ level: in a similar way to the $S M \rightarrow E M$ transition, a site in the $S H$ state evolves to $E H$ according to $p_{e m h}$, the number of $I M$ sites in the $M$ level neighborhood, and the human mobility $\mu$. EH becomes infectious $I H$ after the $H$ virus latent period $t_{l h}$, and $I H$ becomes recovered $R H$ after the viremia period $t_{v}$.

Note that in the above level descriptions, we already included relevant features of dengue transmission mentioned in Sec. I. Seasonal information (rainfall intensity) is used as input data $[6,7]$ by tuning the time dependence of the $f_{s}(t)$ probability using a Fourier expansion of the actual rainfall series. If the time series do not include daily entries or is not complete over the whole simulation period, interpolation or addition of random noise to the day average taken over a few years can be used. Global infection probabilities between $H$ and $M$ populations, due to mobility in private and public transport systems, is described by a global (mean-field) mobility parameter $\mu$. It describes the effect of daily human displacement within the city. The action of $\mu$, which is the same for all sites, is to globally increase the $S M \rightarrow E M$ and $S H \rightarrow E H$ probability transitions, without any influence from the neighborhood population in the other level. Finally, the $M$ level population decreases, which results from vector control actions, is included by the following additional rule: the natural $M$ death probability is increased by an additional amount $p_{a d m}$, which reduces the adult mosquitoes on any state of the $M$ level.

The initial conditions for the CA time evolution are the following: (i) an infection seed, represented by one $I H$ site, is randomly placed in the $H$ level. (ii) A large number of cells in the $S M$ and $E M S$ states is randomly distributed in the $M$ level. (iii) The cells in the $A$ level are randomly distributed into the four possible states.

Some of the basic interaction mechanisms and external effects included in the model have been used, in other contexts, by previous ODE models reported in the literature. Climatic effects have been modeled by seasonal variations in model parameters [24]. Tuning model parameters by comparison to actual data have also been attempted, e.g., by the estimation of the basal transmission rate for age-stratified data from Thailand [25]. Other models have considered the role of a unique vector in the transmission of multiple diseases, as more than one dengue serotype [26-28] or the concurrent transmission of yellow fever in dengue infested areas [29]. Finally, the effects of vector control have already been explicitly analyzed in ODE models $[27,28,30]$.

\section{PARAMETER VALUES}

The CA parameters introduced in the previous section can be classified into four classes, according to the individual
TABLE I. The parameter range of values of temporal parameters and the probabilities of transmission $H-M$ and $M-H$, and of death mosquito according to the literature. The baseline values were chosen for the simulations of the model.

\begin{tabular}{|c|c|}
\hline Parameter & Range of values \\
\hline Egg period $\left(t_{e}\right)[22]$ & 4-5 days \\
\hline Larvae phase period $\left(t_{l}\right)[22]$ & 5-7 days \\
\hline Pupae phase period $\left(t_{p}\right)[22]$ & $2-3$ days \\
\hline $\begin{array}{l}\text { Latent period of virus in the mosquito }\left(t_{l m}\right) \\
{[31,9,22,32]}\end{array}$ & 7-20 days \\
\hline $\begin{array}{l}\text { Latent period of virus in the human }\left(t_{l h}\right) \\
{[9,31,32]}\end{array}$ & $2-12$ days \\
\hline Viremia period $\left(t_{v}\right)[9,31,33]$ & 3-7 days \\
\hline $\begin{array}{l}\text { Probability of transmission human mosquito } \\
\left(p_{\text {ehm }}\right)[34]\end{array}$ & $0.5-1.0$ \\
\hline $\begin{array}{l}\text { Probability of transmission mosquito human } \\
\left(p_{\text {emh }}\right)[9]\end{array}$ & $0.5-1.0$ \\
\hline Probability of mosquito death $\left(p_{m d}\right)[9,29,35]$ & $0.128-0.25$ \\
\hline Lattice size $(L)$ & Variable \\
\hline Mobility parameter $(\mu)$ & Variable \\
\hline
\end{tabular}

processes they describe: (1) spatial parameters: $L$ and $\mu$; (2) temporal parameters: $t_{e}, t_{l}, t_{p}, t_{l m}, t_{v}$, and $t_{l h}$; (3) transmission probability and mosquito death parameters: $p_{e m h}, p_{e h m}$, and $p_{m d} ;$ (4) vector control parameter: $p_{a d m}$.

The values of spatial parameters have been obtained by taking into account the data of a given urban center. We estimate the size $L$ of the lattice (number of sites $=L^{2}$ ) by considering the area of the city $\left(A_{c}\right)$ and the flight radius of the vector $(R)$. More specifically, we assumed that $A_{c}=L^{2} a$, where $a$ is the area of one cell, while $R$ corresponds to the average (Moore) neighborhood radius. This way, we have

$$
R=\frac{\sqrt{a}(1+\sqrt{2})}{2} \Rightarrow L=\sqrt{\frac{A_{c}}{a}}=\frac{\sqrt{A_{c}}(1+\sqrt{2})}{2 R} .
$$

An Aedes aegypti mosquito flies only over a limited region. The diffusion of a mosquito population from the breeding place, where the eggs have been laid, rarely extends itself over a circle of radius $100 \mathrm{~m}$ [36], the value we assume for $R$ in this work. The range of values of $\mu$ was estimated by requiring that the model reproduces the same behavior of the histogram of the number of census tracts with, at least, one reported dengue case during the corresponding time period.

We assumed fixed values (within the range presented in Table I) for the transmission probabilities $p_{\text {ehm }}=p_{\text {emh }}=0.75$ as proposed in [9] and followed in [24,25,27]. The life period of the adult phase vector has been estimated from 4 to 7 days, so that $p_{m d}$ lies in the $[1 / 7,1 / 4]$ interval. For vector control parameter, we scanned the complete interval from 0 to 1 .

Choosing the CA iteration time unit to be 1 day, we are able to set the value intervals for several temporal parameters according to the literature (see Table I). The simulations were performed based on an adaptation of the epidemiological definition of an epidemic process [37] to our model simu- 
lations. A disease outbreak is considered epidemic if the annual incidence $I$, the number of reported cases to susceptible population, is above a certain (epidemic) threshold $I_{t h}$. Therefore, $I_{t h}$ may be given by

$$
I_{t h}=\langle I\rangle+2 \sigma
$$

where the average incidence $\langle I\rangle$ is calculated with respect to the last $N$ years and $\sigma$ corresponds to the standard deviation. To obtain the corresponding model values, we run the program for $N$ different random seeds and evaluate $I_{t h}$ according to definition (2). We recall that, as for actual cases of vectortransmitted diseases, several numerical simulations resulting from different random seeds die out in the first weeks, which is interpreted as small endemic processes. In other words, for model simulations, $I_{t h}$ means a reference value to eliminate samples that are not large enough to be considered as an epidemic process.

After the evaluation of $\langle I\rangle$ and $\sigma$, the program was run as many times as necessary to get $K$-independent samples with $I>I_{t h}$. Although we performed the numerical simulations of the model for large time intervals, our analysis can be restricted to 364 time units if we want to compare the results with the actual data of 1 year epidemic series. The output data are the time series of each state density in the $H, M$, and $A$ levels of the CA model, and the spatial configurations at any time step. The integration CPU time increases with $L^{3}$.

Based on the range of temporal parameter values in Table I, simulations have been conducted for an initial set of parameter values. Then, we investigate the effect of changing one parameter at a time, while holding all the others fixed. This is a simple procedure if compared to other heuristic methods as, for instance, genetic algorithm [38]. Nevertheless, it is enough to reproduce actual time series of different dengue epidemics.

This way, we identified the baseline values that minimize the error between the actual time series and the simulated time series. We performed several tests in order to check the robustness of the chosen initial set of parameter values. For a systematic analysis of parameter values, we considered an average of $M$ simulations samples, identifying the best output for the purpose of comparison with one actual epidemics time series. This is achieved by the analysis of the minimum discrepancy between actual and simulated time series,

$$
e=\frac{\sum_{i=1}^{T}\left|a_{i}-s_{i}\right|}{T},
$$

where $T$ is the number of days, $a_{i}$ is the actual incidence, and $s_{i}$ is the simulated incidence of day $i$. Once the baseline of temporal parameter values was estimated, the analysis of minimal discrepancy was applied to select the best sample in comparison to actual data.

\section{RESULTS}

\section{A. Seasonal effects: Actual and simulated time series}

In order to validate the model, we initially considered the data recorded during the first dengue epidemic in Salvador,

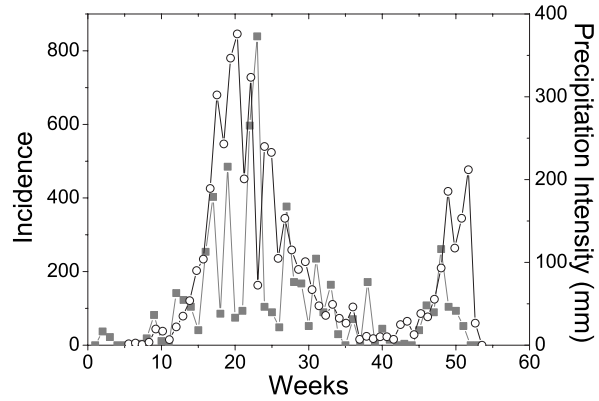

FIG. 3. Time series of the 1995 Salvador weekly rainfall $\Gamma_{R}$ (solid gray squares) and weekly dengue incidence $I_{D}$ (hollow black circles).

Brazil [13], which was caused by one single serotype (DenV2 ). Then to check the robustness of the model we considered the data of another dengue epidemic in another Brazilian city. The event in Salvador occurred in 1995 when its population comprised $p_{c}=2.3$ million habitants. The area of the city amounts to $A_{c}=313 \times 10^{6} \mathrm{~m}^{2}$. The yearly average precipitation is $1980 \mathrm{~mm} / \mathrm{year}$, while seasonal effects concentrate precipitation in the March to August months. The 1995 weekly rain intensity $\Gamma_{R}$ and reported number of new dengue cases $I_{D}$ (incidence) are shown in Fig. 3. The 1995 average daily temperature was $25.89^{\circ} \mathrm{C}$, with 1.47 standard deviation. Since temperature and air humidity are locally very stable, rainfall is the most important climatical factor for dengue propagation. Indeed, the Pearson correlation varies from 0.49 to 0.76 for, respectively, weekly and monthly sampled data. As it will be clear from the discussion of our results, such an increase in the correlation value is due to a roughly two week delay time between the two signals. When the series are clustered in large time windows, such effects become much smaller. The daily rainfall data were provided by the Brazilian National Institute of Meteorology (INMET).

The periodic forcing, represented by the function $f_{s}(t)$, was expressed in terms of the Fourier expansion of the raw data,

$$
f_{s}(t)=a_{0}+\sum_{j=1}^{12} a_{j} \cos (\pi j t / 26)+b_{j} \sin (\pi j t / 26),
$$

where the values of the coefficients are indicated in Table II.

The 10,831 reported dengue cases were georeferenced into 2600 spatial units (census tracts). They were also distributed into 52 time units, called epidemiological weeks [13]. Note that due to large official subnotification and nongeoreferenced cases $(26 \%)$, the actual number of cases is much larger. In Salvador, the epidemic peak occurs before the rainfall peak, which can be justified by the fact that due to the high intense pluviometric precipitation peak, the rainfall washes out the vector in the immature phase. As we will see later, this may not happen in other urban centers. Inserting into Eq. (1) the reported area of Salvador and mosquito flying radius, we are lead to the value $L=214$. To warrant consistency between $L^{2}$ and the population of Salvador, each cell represents 50 individuals. On the other hand, the value $\mu=5 \times 10^{-4}$ has been selected from the interval where the 
TABLE II. Values of the Fourier coefficients of the 1995 Salvador weekly rainfall precipitation series.

\begin{tabular}{rrc}
\hline \hline$n$ & $a_{n}$ & $b_{n}$ \\
\hline 0 & 0.13929 & \\
1 & -0.12878 & 0.04650 \\
2 & 0.06192 & -0.08005 \\
3 & 0.01711 & 0.0831 \\
4 & -0.00424 & -0.04121 \\
5 & 0.00824 & -0.0686 \\
6 & -0.03315 & -0.03859 \\
7 & 0.01013 & 0.0296 \\
8 & -0.04529 & -0.01635 \\
9 & 0.04763 & -0.01109 \\
10 & -0.07674 & 0.04058 \\
11 & 0.04784 & -0.05186 \\
12 & -0.02364 & 0.04138 \\
\hline \hline
\end{tabular}

model is able to reproduce the exponential behavior in the probability distribution of observed new cases in a year among 2600 census tracts (not shown).

Assuming that the presence of just one infected individual in each $H$ lattice site is sufficient to turn it into the $E H$ state, the best sample is able to reproduce the actual data quite well, as shown by the normalized actual and simulated incidence time series in Fig. 4. We normalize the incidence time series just by dividing the corresponding values by the largest number of new weekly cases. Therefore, the corresponding normalization factor for the actual data is $n_{\max } L^{2} / p_{c}$ where $n_{\max }$ is the weekly largest number of new cases in the actual series. In Fig. 4, the normalization factors for the actual and the best simulated time-series incidence are, respectively, 17 and 11 .

To emphasize the importance of the accurate description of the recorded periodic forcing in reproducing the $I_{D}$ values,

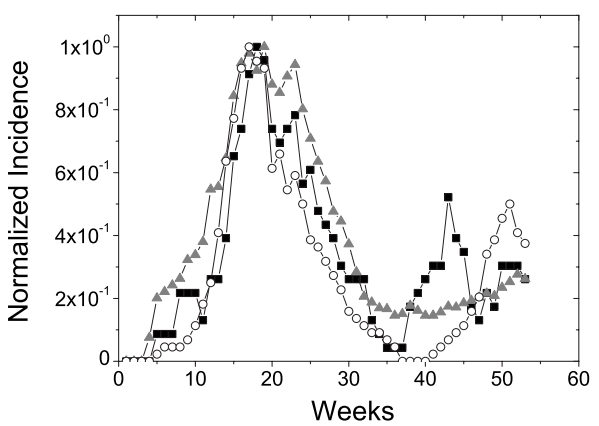

FIG. 4. Actual $\left(I_{D}\right)$ and simulated $\left(I H_{N}\right) 1995$ Salvador weekly incidence time series normalized by the largest single input. The data have been smoothed by averaging on three consecutive weeks. Hollow black circles, solid black squares, and solid gray triangles indicate, respectively, $I_{D}$, the best individual sample, and average value over 20 samples taken from random seeds. The normalization factors for $I_{D}$, the best $I H_{N}$, and the averaged $I H_{N}$ are 17, 11, and 8.14 respectively. The following parameter values have been used: $t_{e}=5, t_{l}=5, t_{p}=3, t_{l m}=7, t_{l h}=6, t_{v}=6, \quad p_{e h m}=p_{e m h}=0.75, \quad p_{m d}$ $=0.143, \mu=5 \times 10^{-4}$, and $L=214$.

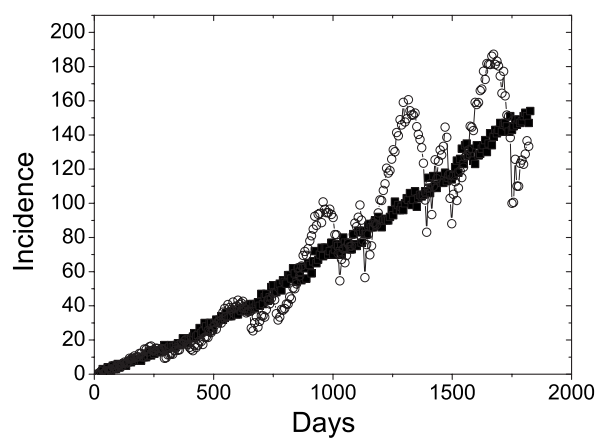

FIG. 5. Average value of simulated weekly incidence $\left(I H_{N}\right)$ when $f_{s}(t)=1$ (solid squares) and $f_{s}(t)=\sin (2 \pi t / 52)$ (hollow circles). The data have been smoothed by averaging on three consecutive weeks. Parameter values are the same as in Fig. 4. Average value taken oven 20 samples.

we draw, in Fig. 5, the time evolution according to two hypothetical scenarios. They were obtained by replacing $f_{s}(t)$, in the first place, by a constant value and afterward by a simple periodic sine function. The resulting incidence counts, which have been extended for a larger time interval (5 years), differ substantially from the typical patterns in Fig. 5. The importance of the accurate description of the external drive, which is a crucial aspect of vector-transmitted diseases, has been neglected in most of the analyzed models with time and space dependence.

The importance of seasonal aspects for the observed dynamics can be further exemplified by running the model with the data of other urban centers. We now consider the 1999 dengue epidemic in Mossoró, in Northeast Brazil [14]. Some features of this outburst differ from those in the Salvador event. For instance, we note that the rainfall peak $\left(\Gamma_{R}\right)$ precedes the reported incidence $\left(I_{D}\right)$ peak. In this case, for which only the monthly data are available for both incidence and rainfall, not only the rainfall regime is different from that in Salvador but also Pearson's correlation coefficient ( $c$ $=0.69$ ) between the rainfall and dengue incidence is smaller (see Fig. 6).

Mossoró's larger surface of $A_{c}=2110 \times 10^{6} \mathrm{~m}$ [39] directly influences spatial parameters, leading to a lattice size

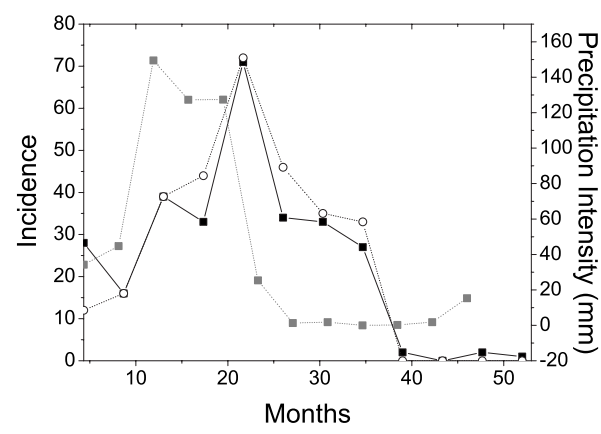

FIG. 6. Time series of the 1999 Mossoró monthly rainfall intensity $\Gamma_{R}$ (solid gray squares), actual dengue incidence $I_{D}$ (hollow black circles), and simulated dengue incidence $I H_{N}$ (solid black squares). The simulated curve corresponds to the best sample. The following parameter values have been used: $t_{e}=4, t_{l}=7, t_{p}=7, t_{l m}$ $=6, t_{l h}=5, t_{v}=6, p_{e h m}=p_{e m h}=0.75, p_{m d}=0.143, \mu=1.0 \times 10^{-6}$, and $L=554$. 


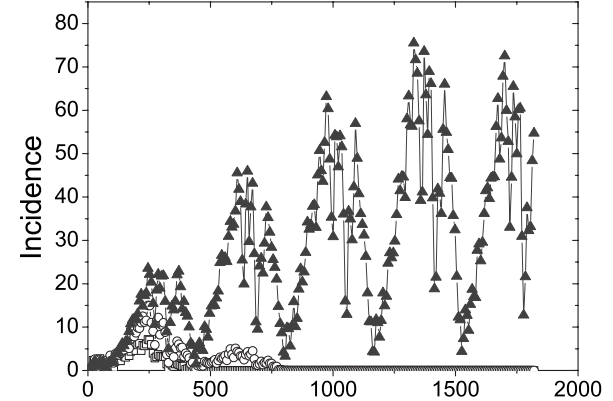

(a)

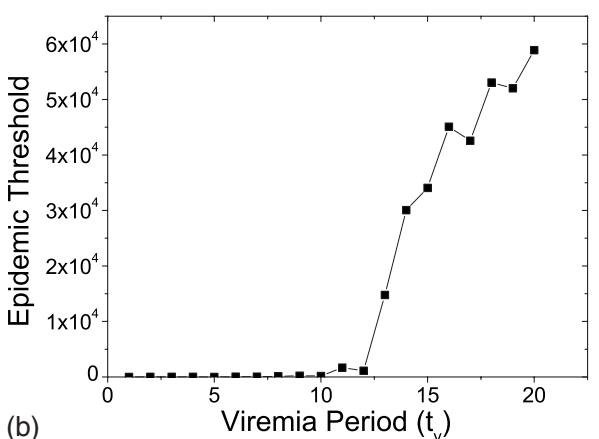

FIG. 7. (a) Predicted average value $\left\langle I H_{N}\right\rangle$ for a large time interval of 5 years as function of human viremia period: hollow squares, hollow circles, and solid triangles indicate, respectively, $t_{v}=6,9$, and 12. Values have not been normalized. Other parameter values are the same as in Fig. 4. Averages have been taken over 20 samples. (b) Dependence of epidemic threshold over 5 years as function of $t_{v}$. A large increase ( $\sim 2$ orders of magnitude) starts at $t_{v} \sim 11$.

of $L=554$. As this incidence data are not georeferenced, $\mu$ could not be directly estimated. However, taking into account that the city is a less developed urban center with a smaller population than Salvador $\left(p_{c}=234.390\right.$ habitants [39]), we consider a smaller value of $\mu=1.0 \times 10^{-6}$. The values of the other parameters were chosen according to the already discussed procedures. The results in Figs. 4 and 6 show that the model is robust enough to simulate dengue incidence for cities with high and low rain intensities, and different Pearson correlation coefficients. Such results indicate that besides the importance of periodic forcing, the epidemic behaviors of vector-transmitted diseases are heavily dependent on entomological and epidemiological aspects that are also caught by the model.

To better understand the forcing effect, the behavior of CA model, with the 1995 Salvador outbreak parameters, has been followed for large time intervals. In this situation, we consider that exactly the same rainfall incidence obtained from 1 year pluviometric data is repeated periodically [12]. Our results indicate that the periodic forcing leads to modulated responses. However, if we disallow the possibility of new exogenous infected sources (due, e.g., to an infected visitor), the amplitude of the epidemic outbursts does not remain the same. If the same parameter values as in Fig. 4 are used, the results in Fig. 7(a) indicate that $I H_{N}$ oscillation amplitude reaches its maximum value in the first year when it starts decreasing in a steady way. It is interesting to note that, at the same time, the $M$ and $A$ populations do not decrease in a similar way. This indicates that in a closed environment, the number of individuals carrying active virus and a relatively weak damping effect due to a small $R H$ population turns it difficult to trigger new epidemic events. This happens in spite of the fact that, after five years, the number of susceptible individuals $S H$ in the population is still very high- $-99.6 \%$ for the same parameter set.

On the other hand, Fig. 7(a) also shows that changes in the parameter values, favoring virus permanence in the $H$ level for a longer time, may lead to the opposite landscape, with a long period during which the yearly amplitude of the $I H_{N}$ population increases monotonically. In such cases, the amplitude decreases only when a large fraction of the $H$ population has become infected and switched to the $R H$ state. Note that this is not yet the situation, after a 5 year evolution period, for such alternative time evolution scenarios. There, we still find a large fraction of $\mathrm{SH}$ susceptible individuals, $84.3 \%$, where the variation occurs in the human viremia $\left(t_{v}=12\right)$.

The size of successive epidemic events in isolated environments depends on the value of $t_{v}$ in a nonlinear way. As long as such values are inside the observed range (and even $\sim 40 \%$ larger), the obtained patterns predict a decrease in the size of subsequent yearly events. However, when this parameter becomes much larger (in Fig. 7(a), $t_{v}=12$, 70\% larger than upper bound $\sim 7$ days), the predicted pattern changes markedly. In Fig. 7(b), such a dependence is synthesized by drawing the epidemic threshold as function of $t_{v}$. These results both confirm the structural stability of the model for parameter values inside the confidence range, as well as its ability to predict new possible scenarios beyond the parameter bounds in Table I. As far as we know, this effect, resulting from a local interaction among the three CA levels, has not been previously discussed in the literature.

\section{B. Mobility effects: Spatiotemporal patterns and vector control}

Spatiotemporal patterns resulting from georeferenced data of the actual epidemics of Salvador in 1995 have been reported elsewhere [13]. They can be compared to the CA simulated spatiotemporal patterns, which have been generated with the help of the G2 graphic package [40]. We have found that the model is able to qualitatively reproduce the main features observed in actual spatiotemporal epidemic patterns [13].

In Fig. 8, we illustrate spatiotemporal patterns for $A, M$, and $H$ populations in some characteristic time steps. For the sake of a better visualization, we choose a small value of lattice size $(L=79)$. Using the same initial conditions presented in Sec. II, the simulations show that epidemics start around the site where the $I H$ seed was located. Neighboring $S M$ cells change into $E M$ state, disseminating the disease into other $H$ sites, while increasing the radius of the primary epicenter. Due to $H$ and $M$ mobilities, some secondary epicenters are formed. In this case, without any control strategy, the epidemics evolve naturally until its end. Figure 8 reveals qualitative similarities to the main features presented in [13]: 


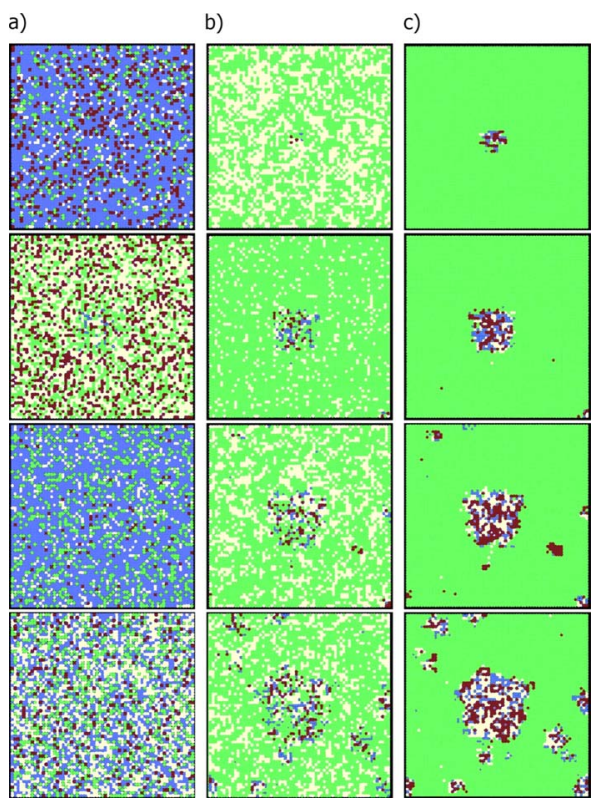

FIG. 8. (Color online) Simulated spatiotemporal configurations of cumulated cases for the following parameter values: $L=79$, $p_{m d}=0.143 p_{\text {emh }}=p_{\text {ehm }}=0.75 \mu=0.001, t_{e}=5, t_{l}=7, t_{p}=3, t_{l m}=7$, $t_{l h}=5$, and $t_{v}=5$. Four snap shots are shown for each lattice: (a) aquatic phase $(A)$; (b) mosquito $(M)$; and (c) human $(H)$. Online version: $(A)$ —empty site: light yellow; $E$ : green; $L / P$ : blue; $B$ : dark red; $(M)$ —empty site: light yellow; $S M$ : green; $E M$ : blue; $I M$ : dark red; $(H)-S H$ : green; $E H$ : blue; $I H$ : dark red; $R H$ : light yellow). Printed version: $(A)$ —empty site: white; $E$ : light gray; $L / P$ : gray; $B$ : dark gray; $(M)$ —empty site: white; $S M$ : light gray; $E M$ : gray; $I M$ : dark gray; $(H)-S H$ : light gray; $E H$ : gray; $I H$ : dark gray; $R H$ : white.

the persistence of the primary epidemic epicenter, the emergence of secondary epicenters, and an irregular shape of each epicenter.

Secondary epicenters at large distances from the original seed are a direct consequence of the mobility effects, which are well accepted to be an important feature for dengue transmission in urban centers. Indeed, if $\mu=0$, the shown spatiotemporal pattern is replaced by a diffusionlike pattern with a single epicenter. However, $\mu$ also plays an important role in reducing relative fluctuations, an expected "mean-fieldlike" effect. Such an effect is particularly intense for large values of incidence, as shown in Fig. 9. On the other hand, Fig. 9 also illustrates that very small values of $\mu$ have a large influence in the epidemic process, producing an increase in 2 orders of magnitude on the outbreak intensity.

Until today, no efficient vaccine against dengue could be devised. Therefore, actions toward vector control constitute the only public health policy to reduce the deleterious effect of the disease. Even so, there are still controversies regarding whether vector control actions are more reliable in the $A$ or $M$ phases. As the CA model is able to successfully reproduce epidemic data and follow the dynamics of the disease for longer periods of time, it can also provide useful insights regarding the efficiency of vector control actions.

To this purpose, let us consider the dependence between the epidemic threshold and the mature phase vector control

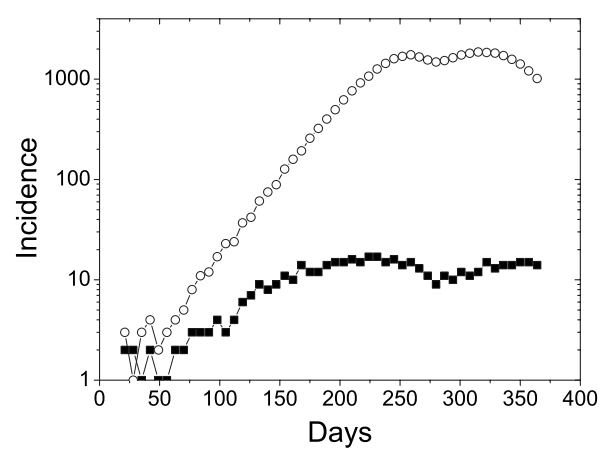

FIG. 9. Illustration of the mobility parameter effect. Small nonzero value of $\mu$ largely increases the event sizes. The average value of $I H_{N}$ are taken over 200 samples. Parameter values are the same as in Fig. 4, with the exception of $\mu$ that assumes the following values: 0.0 (solid black square) and 0.02 (hollow black circle). For the sake of a better comparison between events of rather distinct magnitudes, logarithmic scale is used on the vertical axis.

parameter $p_{a m d}$. We have performed a large number of independent simulations for different values of $p_{a m d}$. We evaluated $I_{t h}$ with the help of Eq. (2), where the time average was replaced by sample averages. Since the epidemic intensity is as strong as large the value of $I_{t h}$, this parameter measures the probability that an individual living the simulated urban center gets infected within 1 year.

The results in Fig. 10 show that when $\mu=0, I_{t h}$ is almost insensitive to $p_{\text {amd }}$ as long as $p_{\text {amd }}<0.01$. For larger values, the plot shows an expressive decrease in the value of $I_{t h}$. Although the pattern suggests a power-law behavior, this effect is valid only over a short interval ( $\sim 1$ decade). The reduction in the value of $I_{t h}$ shows that effective policies aiming at a reduction in the vector reproduction in its own environment produce substantial reduction in affected population. This effect is still more expressive and relevant when we consider more realistic situations, in which human and vector population move in the urban space. As expected, the epidemic threshold is larger, for any value of $p_{\text {amd }}$, when $\mu$ $>0$ than when $\mu=0$. Besides that, when $\mu>0, I_{\text {th }}$ decays with respect to $p_{\text {amd }}$ in a faster way in comparison to the $\mu$

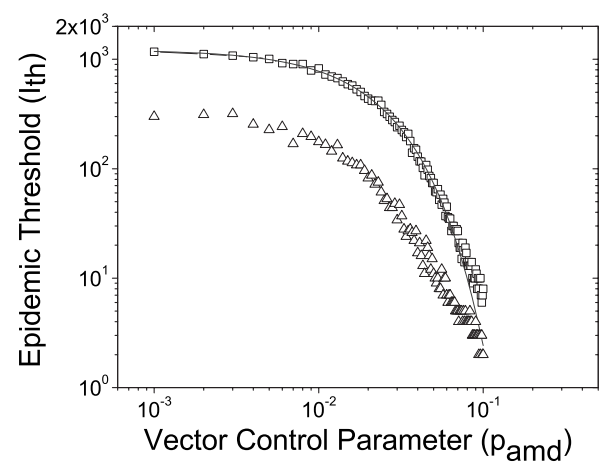

FIG. 10. Illustration of vector control actions. The simulated $I_{t h}$ is drawn as function of vector control parameter $\left(p_{a m d}\right)$. We consider 20 samples and the same parameter values of Fig. 4, except for $\mu=0$ (triangles) and $\mu=10^{-5}$ (squares). The stretched exponential fitting values (solid line) are $b_{1}=(1.218 \pm 0.007) \times 10^{3}, b_{2}$ $=86.4 \pm 4.0$, and $\beta=1.14 \pm 0.01$. 
$=0$ situation. As shown in Fig. 10, the points fit quite well to a stretched exponential $I_{t h}=b_{1} \exp \left[-b_{2}\left(p_{a m d}^{\beta}\right)\right]$.

\section{CONCLUDING REMARKS AND PERSPECTIVES}

The three-level CA model investigated in this work has been proven successful in quantitatively reproducing actual time series of dengue epidemics. As such, the CA main assumptions can be extended or adapted to the description of similar vector-transmitted diseases. Besides the usual local interaction steps based on SEIR compartment models, the most important novelties are (i) the use of multiple levels to describe the relevant populations involved in the disease spreading; (ii) the use of the climatical data as input data; (iii) the $A-M$ and $M-H$ interlevel interactions; (iv) the inclusion of short-range vector mobility and long-range human mobility.

The model is robust with respect to the range of parameters considered in the literature and to its ability in reproducing time series of dengue epidemics in different urban centers. The climatic input data, as well as the procedure used for estimating the parameter values, are able to catch the diversity of the dengue incidence time series for different cities. Although we have mainly focused our analysis on the human population, the CA model also provides useful insights on the behavior of the vector population, which will be detailed discussed in a future work.

The analysis of vector control shows that, as expected, it indeed produces a decrease in the probability of human in- fection. However, we have shown that this effect is more relevant when vector and human mobility are taken into account. In this case, the infection probability decreases according to a stretched exponential, while a power-law behavior is observed when the no mobility assumption is taken into account.

Once the basic dynamical aspects of the model have been validated, perspectives for further work on this model are of two kinds. The first one amounts to investigate the impact of different strategies of vector control on dengue transmission as well as to discuss the detailed behavior of $M$ and $A$ populations subject to those strategies. A more ambitious goal is to achieve the quantitative reproduction of spatial patterns. This requires a more precise local characterization of spatial units, as well as global positioning system georeferencing data. This way, the CA model can help in planning improved vector control policies from the spatial point of view, attacking mainly the most important foci for the epidemic triggering.

\section{ACKNOWLEDGMENTS}

The authors thank C. P. Ferreira, D. Alves, E. Massad, H. M. Yang, J. G. V. Miranda, J. P. Dias, L. Esteva, M. N. Burattini, and V. C. G. S. Morato for useful discussions about dengue modeling. The authors acknowledge the Brazilian agencies CNPq and FAPESB for financial support; and INMET for rainfall data.
[1] N. Boccara, Modeling Complex Systems Series: Graduate Texts in Contemporary Physics (Springer Verlag, New York, 2004).

[2] P. Philippe, Nonlinear Dyn. Psychol. Life Sci. 4, 275 (2000).

[3] R. Anderson and R. May, Infectious Diseases of HumansDynamics and Control (Oxford University Press, Oxford, 1991).

[4] G. Chowell, J. M. Hyman, S. Eubank, and C. Castillo-Chavez, Phys. Rev. E 68, 066102 (2003).

[5] D. J. Gubler, in Dengue and Dengue Hemorrhagic Fever, edited by D. J. Gubler and G. Kuno (CAB, New York, 1997), pp. $1-22$.

[6] S. Hales, N. de Wet, J. Maindonald, and A. Woodward, Lancet 360, 830 (2002).

[7] G. Kuno, Epidemiol. Rev. 17, 321 (1995).

[8] World Health Organization, 2008, http://www.who.int/ mediacentre/factsheets/fs117/en/

[9] E. A. C. Newton and P. Reiter, Am. J. Trop. Med. Hyg. 47, 709 (1992).

[10] N. A. Maidana and H. M. Yang, Tend. Mat. Apl. Comput. 8, 83 (2007).

[11] C. P. Ferreira, P. Pulino, L. T. Takahashi, and H. M. Yang, Math. Popul. Stud. 13, 215 (2006).

[12] A. Vecchio, L. Primavera, and V. Carbone, Phys. Rev. E 73, 031913 (2006).

[13] F. R. Barreto, M. G. Teixeira, M. C. N. Costa, M. S. Carvalho, and M. L. Barreto, BMC Public Health 8, 51 (2008).

[14] J. P. Dias, Ph.D. thesis, Universidade Federal da Bahia, Brazil, 2006.

[15] D. A. T. Cummings, R. A. Irizarry, N. E. Huang, T. P. Endy, A. Nisafaix, K. Ungchusak, and D. S. Burge, Nature (London) 427, 344 (2004).

[16] R. M. Zorzenon dos Santos and S. G. Coutinho, Phys. Rev. Lett. 87, 168102 (2001).

[17] R. M. Zorzenon dos Santos, S. T. R. Pinho, C. P. Ferreira, and P. C. A. da Silva, Eur. Phys. J. Spec. Top. 143, 125 (2007).

[18] E. A. Reis, L. B. L. Santos, and S. T. R. Pinho, Physica A 388, 1303 (2009).

[19] A. Johansen, J. Theor. Biol. 178, 45 (1996).

[20] S. L. Silva, J. A. Ferreira, and M. L. Martins, Physica A 377, 689 (2007).

[21] S. C. Ferreira and M. L. Martins, Phys. Rev. E 76, 036112 (2007).

[22] N. A. Honório, W. C. Silva, P. J. Leite, J. M. Gonçalves, L. P. Lounibos, and R. Lourenço-de-Oliveira, Mem. Inst. Oswaldo Cruz 98, 191 (2003).

[23] M. G. Teixeira, M. C. N. Costa, M. L. Barreto, and L. E. Mota, Cad Saude Publica 21, 1307 (2005) FIOCRUZ.

[24] F. A. B. Coutinho, M. N. Burattini, L. F. Lopez, and E. Massad, Bull. Math. Biol. 68, 2263 (2006).

[25] N. M. Ferguson, C. A. Donnelly, and R. M. Anderson, Philos. Trans. R. Soc. London, Ser. B 354, 757 (1999b). 
[26] L. Esteva and C. Vargas, J. Math. Biol. 46, 31 (2003).

[27] L. M. Hartley, C. A. Donnelly, and G. P. Garnett, Trans. R. Soc. Trop. Med. Hyg. 96, 387 (2002).

[28] I. B. Schwartz, L. B. Shaw, D. A. T. Cummings, L. Billings, and M. McCrary, and D. S. Burke, Phys. Rev. E 72, 066201 (2005).

[29] E. Massad, F. A. B. Coutinho, M. N. Burattini, and L. F. Lopez, Trans. R. Soc. Trop. Med. Hyg. 95, 370 (2001).

[30] L. Esteva and H. M. Yang, Math. Biosci. 198, 132 (2005).

[31] H. M. Yang and C. P. Ferreira, Appl. Math. Comput. 198, 401 (2008).

[32] J. F. Siler, M. W. Hall, and A. P. Hitchens, Philippine. J. Sci. 29, 1 (1926).

[33] D. J. Gubler, R. T. Suharyono, R. Tan, M. Abidin, and A. Sie,
Bull. World Health Organ. 59, 623 (1981).

[34] L. Rosen, L. F. Roseboom, D. J. Gubler, J. C. Lien, and B. N. Chaniotis, Am. J. Trop. Med. Hyg. 34, 603 (1985).

[35] P. M. Sheppard, W. W. MacDonald, R. J. Tonn, and B. Grab, J. Anim. Ecol. 38, 661 (1969).

[36] C. Liew and C. F. Curtis, Med. Vet. Entomol. 18, 351 (2004).

[37] D. P. Barker and F. J. Bennett, Practical of Epidemiology (Churchill Livingstone, Edinburgh, 1976).

[38] M. Mitchell, An Introduction to Genetic Algorithms (MIT Press, Cambridge, MA, 1996).

[39] BRASIL, Instituto Brasileiro de Geografia e Estatística (IBGE), 2008, http://www.ibge.gov.br/home/

[40] Lj. Milanovic and H. Wagner, g2-graphic library (C), 1999, http://g2.sourceforge.net 